\title{
Cognitive Function-Abilities
}

National Cancer Institute

\section{Source}

National Cancer Institute. Cognitive Function-Abilities. NCI Thesaurus. Code C121995.

A patient reported outcome instrument that assesses patient-perceived functional abilities related to cog nitive tasks. 